\title{
Prediction of the Evolution of the Surface Roughness in Dependence of the Lubrication System for Cold Forming Processes
}

\author{
Peter Groche $^{1} \cdot$ Philipp Kramer $^{1} \cdot$ Sebastian Zang ${ }^{1} \cdot$ Vladimir Rezanov $^{1}$
}

Received: 18 December 2014/ Accepted: 10 April 2015/Published online: 23 May 2015

(c) The Author(s) 2015. This article is published with open access at Springerlink.com

\begin{abstract}
Lubricants for cold forging operations do not only enable a reliable forging process, but also affect the results of forging processes. A sound lubrication decreases the occurring forces and may result in a smooth specimen surface. However, some lubricants are known to increase the initial surface roughness of the specimen as a consequence of the forming. Therefore, the decision which lubricant should be used is of special interest for near-net shape operations. The paper at hand presents the results of a finite element investigation of the first stage of an industrial rod extrusion process which was investigated with two different lubrication systems. The numerical investigations focus on a reliable prediction of the development of the surface roughness in due consideration of the lubricant.
\end{abstract}

Keywords Surface roughness - Cold forging - Rod extrusion $\cdot$ FEA $\cdot$ Sliding compression test $\cdot$ Lubrication system

\section{Introduction}

Cold forging processes are utilized to produce components in large numbers with (near) net shape geometries and improved mechanical properties compared to machined components. Particularly when producing net shape components, the surface quality is an important factor when determining the overall quality of the component. The surface quality of formed components is influenced by

Philipp Kramer

kramer@ptu.tu-darmstadt.de

1 Institute for Production Engineering and Forming Machines, Technische Universitaet Darmstadt, Darmstadt, Germany numerous parameters, such as the initial surface roughness, the relative sliding speed, the temperature within the contact zone, the sliding distance, the contact normal stresses and the lubrication system employed [1]. Among these, lubrication is one parameter that can actively be modified to suit the component specifications, since geometrical variations as well as the material are often a set constraint. However, the selection of a lubrication system is constrained by high tribological loads which are typical for cold forming [2-5]. In addition, the use of a specific lubrication system again determines the resulting surface roughness of the produced part. Although the finite element analysis (FEA) has evolved into a powerful tool in terms of designing forming processes, the modeling of the surface evolution is not yet possible in commercially available FE software. Thus, the process design in terms of surface quality depends heavily on the experience of the process designer.

In addition to process design, it is known that friction between work piece and tool is, among other influencing factors, a dependency of the surface roughness [1]. Kudo [6] suggested in his theoretical work that lubricant trapped between surface asperities can influence the friction in between contact partners. Hu et al. [7] studied the relationship between friction and surface topography in ring upsetting tests and rod extrusion operations for different lubricants and initial work piece surface topographies. Smooth surfaces created by shot blasting were found to reduce the friction. Jung et al. [8] found in downsized tip tests that the friction factor increases with an increase in the surface roughness.

Cristino et al. [9] and Sahin et al. [10] both investigated the effect of changes in surface roughness in regard to the friction coefficient in ring upsetting tests with varying 
tribological pairs. It was found that friction is dependent on changes in the surface roughness.

To mathematically describe technical surfaces, different approaches have been pursued. Greenwood [11] introduced a physical approach which assumes the surface to consist of hemispherical shapes. Building upon this theory, Hol et al. [12] have developed a surface evolution model for sheet metal forming. On the other hand, numerical modeling has been employed to describe the asperity deformation by Korzewka et al. [13]. Kießling presented a 3DFE-Model approach for examining friction based on real surface topography data [14]. However, these numerically based approaches require a highly detailed finite element mesh and are thus very resource intensive. Therefore, these models cannot be utilized for a better calculation of the friction coefficient, since the calculation times would be too high.

Recently, Stahlmann et al. [15] presented a phenomenological model to describe the surface evolution for cold forming processes. With the help of sliding compression tests (SCT), the surface evolution of the specimens was determined and the necessary model parameters were thus obtained. It was shown that with the proposed model, the surface evolution in terms of the surface roughness $S q$ can be predicted with a high quality for cold formed work pieces with surfaces that were initially deterministically structured by rotary swaging.

\section{Aim and Approach}

It has been shown that it is possible to model the surface evolution of deterministically structured bar stock with a tribological system consisting of zinc phosphate $(\mathrm{ZnP})$, sodium soap and oil in a laboratory environment [15]. Within this current study, it will be shown that the developed surface evolution model is also applicable to nondeterministically structured surfaces with state-of-the-art multiple layer lubrication systems. In addition, it will be illustrated that the model is able to predict quantitative differences of the surface evolution in between different tribological systems.

In Fig. 1, the steps performed to achieve this are depicted as well as the corresponding inputs and outputs. After preparation (1) (see Sect. 3.1) of the specimens for the SCT as well as rod extrusion process, the SCT is performed (2) (compare Sect. 3.2). The formed specimens are examined via confocal white light microscopy (3) to determine the surface roughness (see Sect. 3.1). Then, the model parameters can be calculated with a least squares fit (4) (compare Sect. 3.4). The model parameters are then used as input for the numerical simulation (5) (see Sect. 3.4), which is used to calculate the numerical surface

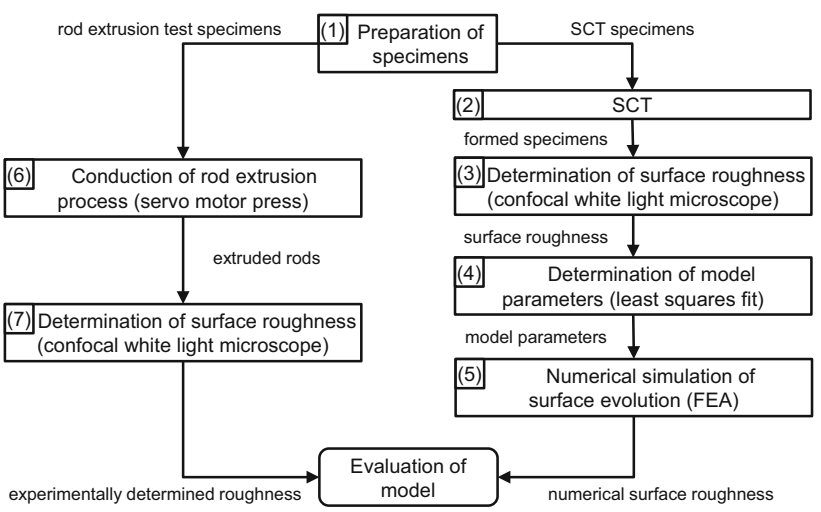

Fig. 1 Flowchart of the study

roughness of the rod extrusion process. The rod extrusion specimens are formed with the help of a servo motor press (6) (compare Sect. 3.3), and the extruded rods are microscopically analyzed (7) to determine the surface roughness (compare Sect. 3.1).

The performance of the model will be evaluated by comparison with the numerical prediction and experimental investigation.

\section{Experimental Setup}

\subsection{Specimen Preparation and Characterization}

Figure 2 depicts the process of specimen preparation for the SCT as well as the rod extrusion process. Two different lubrication systems were investigated; both consisted of a $\mathrm{ZnP}$ conversion coating. For lubrication, a $\mathrm{MoS}_{2}$ coating and a salt-wax coating were chosen. All specimens consisted of $16 \mathrm{MnCrS5}$ (1.7139).

The test specimens were blasted to ensure a comparable surface roughness as well as surface morphology. The surfaces of both the rod extrusion process and the sliding compression specimens were blasted with blasting abrasive F20/S390 from Ferrosad to adjust the initial surface roughness to $\mathrm{Sq}_{\text {rod }}=7.8 \pm 0.7 \mu \mathrm{m}$ and $\mathrm{Sq}_{\mathrm{SCT}}=6.8 \pm 0.4 \mu \mathrm{m}$. After blasting, specimens were coated with a $\mathrm{ZnP}$ conversion

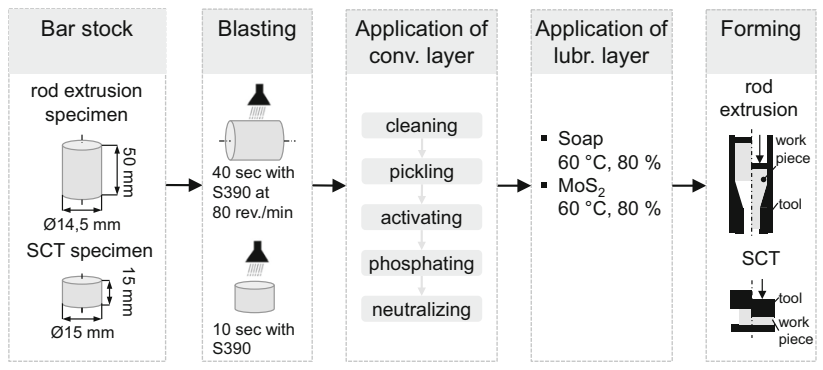

Fig. 2 Specimen preparation 
layer. Then, all specimens were coated with a $80 \mathrm{wt} \%$ solution of $\mathrm{MoS}_{2}$ or salt-wax. From here onwards, the lubrication system $\mathrm{ZnP}+\mathrm{MoS}_{2}$ and $\mathrm{ZnP}+$ salt-wax are referred to as $\mathrm{MoS}_{2}$ and salt-wax (if subscripted, s-w), respectively. After coating, the surface roughness of the specimens was measured at $\mathrm{Sq}_{\mathrm{MoS} 2}=3.4 \pm 0.6 \mu \mathrm{m}$ and $\mathrm{Sq}_{s-w}=3.8 \pm 1.1 \mu \mathrm{m}$. Specimens with a diameter of $d_{\mathrm{SCT}}=15 \mathrm{~mm}$ and a height of $h_{\mathrm{SCT}}=15 \mathrm{~mm}$ were used to carry out the SCTs. Specimens with a diameter of $d_{\mathrm{RE}}=14.5 \mathrm{~mm}$ and a height of $h_{\mathrm{RE}}=50 \mathrm{~mm}$ were used to carry out the rod extrusion process. To obtain the material characteristics for the numerical simulation, flow curves were determined with a Zwick + Roell Z100 testing machine according to specifications by the German Cold Forging Group [16]. The specimens were obtained from the same material charge and turned to a diameter of $d=10 \mathrm{~mm}$ and a height of $h=12 \mathrm{~mm}$. According to these guidelines, the height/diameter ratio can vary from 1.2 to 2.0. However, all specimens with a ratio above 1.2 were asymmetrical after upsetting. Therefore, the mentioned geometry $(d=10 \mathrm{~mm}$, $h=12 \mathrm{~mm}$ ) was chosen. To reduce friction to a minimum, the faces of the specimens were ground to a roughness $\mathrm{Sq}<0.1 \mu \mathrm{m}$ and a $50-\mu \mathrm{m}$-thick Teflon foil was used as lubrication at both faces during upsetting. Flow curves were obtained at room temperature for strain rates of $0.1-0.2 \mathrm{~s}^{-1}$, see Fig. 3. The experiments were repeated three times each. Initial plastic flow was identified at $507 \mathrm{~N} / \mathrm{mm}^{2}$. Due to the force limitation of $100 \mathrm{kN}$ of the testing machine, flow curves could only be determined up to a true strain of $\varphi=0.45$. For the FEA, the flow stress curve was extrapolated up to a true strain of 1 with the help of the power law in the form of

$f_{k}=A \cdot \phi^{B}$

with $A=819$ and $B=0.1$. The power law was fitted to the flow curve within the interval of true strain of $0.3<\varphi<0.45$ with a mean square error of the residuals mse $<0.01$.

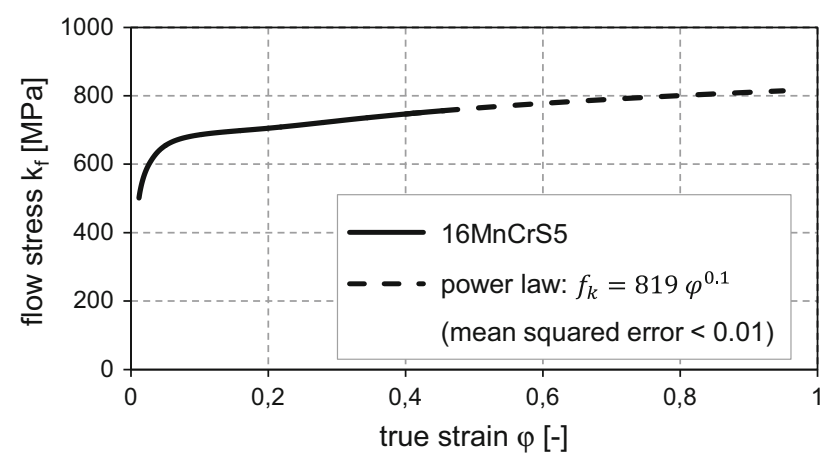

Fig. 3 Measured and interpolated flow curve
The confocal white light microscope $\mu$ Surf from NanoFocus was used to determine the surface topography. All measurements were performed with an objective lens from Olympus with $20 \times$ magnification. Measurements of specimens from the rod extrusion process were performed along the outline of the extruded specimen diameter, see Fig. 4a. Each measurement comprised a measuring field of $800 \times 800 \mu \mathrm{m}^{2}$. For the determination of the resulting roughness of the sliding compression test, multiple measurements of the specimen surface were performed, see Fig. 4b. In total, four measurements were taken per specimen, with each measurement comprising a measuring field of $1460 \times 1460 \mu \mathrm{m}^{2}$.

For analyzing the surface data, the software MountainsMap Expert from Digitalsurf was used to derive the surface roughness $\mathrm{Sq}_{m}$. To compensate skewness of the measured field, the measured data were adjusted with a linear (for the face surface of the cylinder specimen) or a polynomial (for the curved surface of the cylinder specimens) fitting function.

\subsection{Sliding Compression Test}

The determination of the surface evolution during cold forming processes was conducted with the SCT. This tribological test stand was developed at the Institute for Production Engineering and Forming Machines (PtU) [17]. A dual acting hydraulic press is utilized to apply the necessary contact normal stresses of up to $3000 \mathrm{~N} / \mathrm{mm}^{2}$ and surface enlargements of up to 11 by compressing the specimen. For reproducing the acting relative velocities, which can amount to $500 \mathrm{~mm} / \mathrm{s}$ during cold forging operations, the compression plate is slid in relative motion against the specimen while the compression force is maintained, see Fig. 5.

All experiments in this study were conducted with a number of three specimens per series. The specification for the SCT is given in Table 1.

(a)

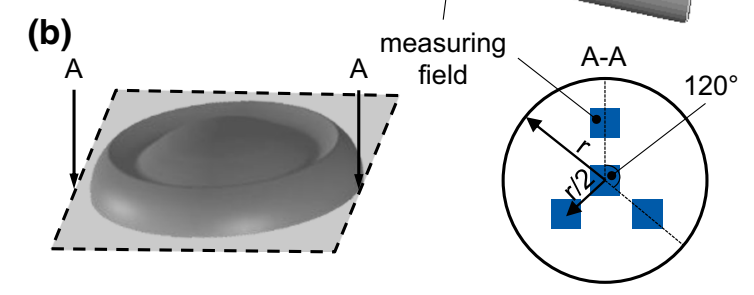

Fig. 4 Measuring fields on a rod extrusion and b SCT specimens 

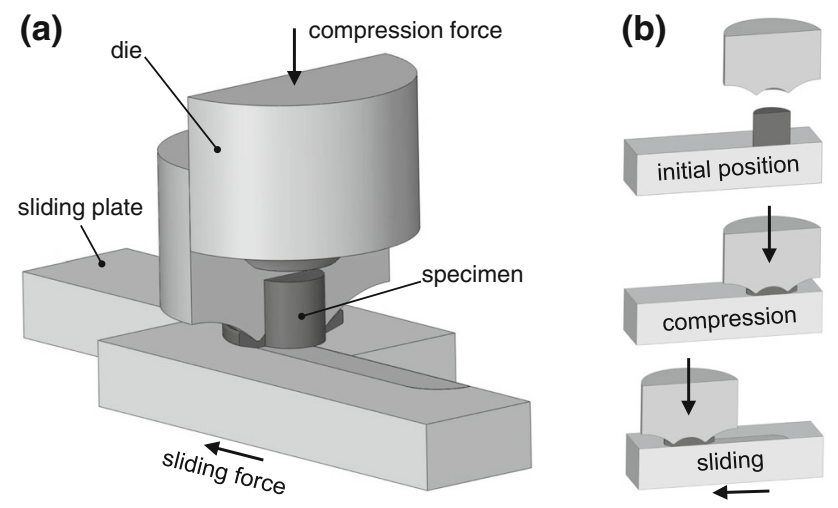

Fig. 5 Sliding compression test (SCT)

\subsection{Rod Extrusion Process}

The first stage of an industrial rod extrusion process for the production of bolts was used to verify the predictions of the FEA. The process specifications are given in Table 2. The specimen was prepared as depicted and described in Fig. 2. The forming process was carried out with a 250-t directdriven servo motor press SWP 2500 from SynchroPress. Both experimental forming series were reproduced three times. After each series, the tool was cleaned.

\subsection{Numerical Setup}

For conducting the FEA, Simufact version 11 was utilized. The rod extrusion process was modeled axisymmetric with a four-node element type and four integration points. The mesh was discretized with an initial edge length of $0.1 \mathrm{~mm}$. The tools were modeled as rigid bodies. The SCT was modeled with a four-node element type and initial edge length $0.4 \mathrm{~mm}$ with Coulomb's law of friction with a constant friction coefficient of $\mu=0.00, \mu=0.04$ and $\mu=0.08$. These simulations were used to determine the loading cases for the parameterization of the friction model. The FEA of the SCT was compared to experimental results in order to ensure its viability, see Fig. 6.

For both simulations, an adaptive global remeshing criterion, dependent on element strain and work piece penetration, was used.

To describe the evolution of the surface, the three-dimensional root mean square $\mathrm{Sq}$ of the profile was used [18], see Eq. (2),

Table 1 Experimental details for the SCT
Table 2 Details of the rod extrusion process

\begin{tabular}{lc}
\hline Parameter & Value \\
\hline Specimen material & $16 \mathrm{MnCrS5}(1.7139)$ \\
Stroke; stroke velocity & $40 \mathrm{~mm} ; 28 \mathrm{~mm} / \mathrm{s}$ \\
Diameter reduction & 0.26 \\
\hline
\end{tabular}

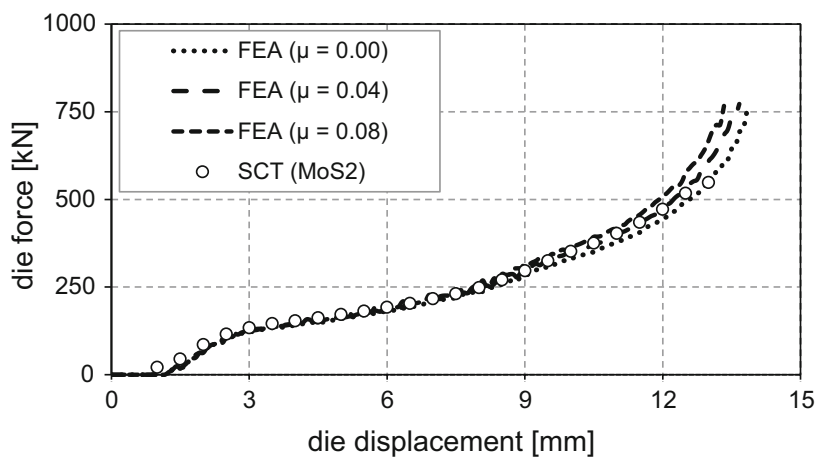

Fig. 6 Comparison of force-displacement curves of the SCT

$\mathrm{Sq}=\sqrt{\frac{1}{A} \iint z^{2}(x, y) \mathrm{d} x \mathrm{~d} y}$

with $A$ constituting the measured field, $x$ and $y$ being the coordinates of the digitized surface and $z$ representing the height of the measured surface.

According to [15], the evolution of the surface roughness can be described by its incremental change

$\mathrm{Sq}_{i}=\mathrm{Sq}_{i-1}-\mathrm{dSq}$

with $\mathrm{dSq}$ being the incremental change of the surface roughness between two states and $\mathrm{Sq}_{i-1}$ being the surface roughness of the preceding increment. The incremental roughness change is in turn determined with Eq. (4):

$\mathrm{dSq}=\left(\mathrm{Sq}_{i-1}-\mathrm{Sq}_{\text {tool }}\right)\left(p_{1} \mathrm{~d} \psi+p_{2} \mathrm{~d} \frac{\sigma_{n}}{k_{f}}\right)$

with $\mathrm{d} \psi=\mathrm{d} A_{I} / A_{O}$ describing the incremental change of the surface enlargement and $\mathrm{d}\left(\sigma_{n} / k_{f}\right)$ describing the incremental change in the normalized contact normal pressure, $p_{1}$ and $p_{2}$ being dimensionless parameters that allow for an alignment of the surface evolution model to the forming environment, e.g., the employed tribological system, the material combination and surface morphology. $\mathrm{Sq}_{\text {tool }}$ denominates the roughness of the employed tools.

For application of the surface evolution model, parameters $p_{1}$ and $p_{2}$ are determined with the help of the sliding compression test. Test runs with different process loads are performed, and the change in roughness is determined with the help of confocal white light microscopy. Parameters $p_{1}$ and $p_{2}$ are then identified by a least squares fit, as depicted in Eq. (5) [15]. 
$\min =\sum\left(\mathrm{Sq}\left(\psi, \frac{\sigma_{n}}{k_{f}}, p_{1,2}\right)-\mathrm{Sq}_{m}\right)^{2}$

For implementation of the surface evolution model within FEA, subroutines were used. The implementation of the surface evolution model is described in the flowchart in Fig. 7.

In contrast to Stahlmann et al.'s implementation [15], the surface data were not stored in an additional background mesh, e.g., matrix. Within Simufact, it is possible to store user-defined data (up to 20 data sets) alongside other nodal data (such as coordinates or contact stress). These datasets were used to store the surface roughness Sq. This approach of using the available user variables has the advantage that the surface data are not mapped to the additional background mesh at every increment step since they are stored alongside the nodal data. This results in less of an information loss, since the surface data is only remeshed and mapped during global remeshings, while the data stored on a background mesh are mapped at every increment step. For further details, see [15].

However, a distortion of the surface data is observed during remeshing. This is due to the fact that during remeshing, the weighted average of nodal data from adjacent nodes is computed. This can lead to miscalculations in regard to the data stored in the user variables when nodes from the inner volume of the work piece are taken into account, since these contain no data relating to the surface. Therefore, if a remeshing has been detected in between increments, user variables are interpolated by means of a subroutine after the start of a new increment. For every node of the newly created mesh, two nodes with the lowest distance are identified with the help of a linear search algorithm. Then, surface values are computed based on an inverse distance weighted algorithm, see Eq. (6) and for explanation see Fig. 8.

$\mathrm{Sq}_{n_{l}}^{h+1}=\sum w_{i} \mathrm{Sq}_{n_{i}}^{h}=\frac{\sum \frac{\mathrm{Sq}_{i}^{h}}{d_{i}^{p}}}{\sum \frac{1}{d_{i}^{p}}}$

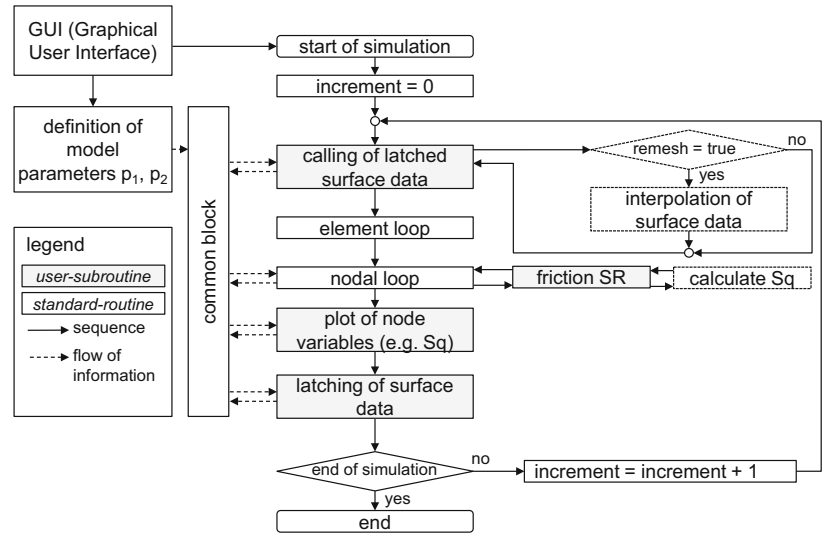

Fig. 7 Flowchart of (sub-)routines

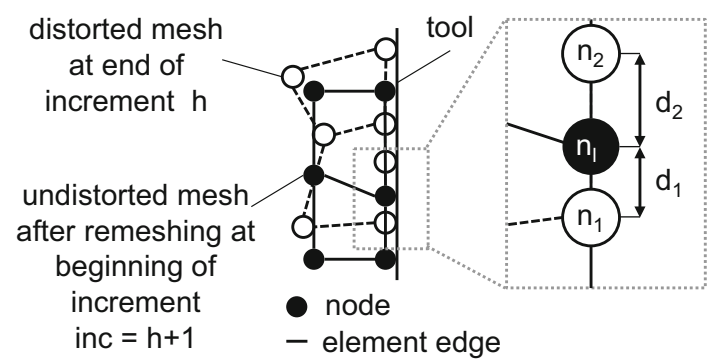

Fig. 8 Denominations for mapping of data

$\mathrm{Sq}_{n_{l}}^{h+1}$ here stands for the interpolated value of the newly created node $l$ at increment $h+1, d_{i}$ being the distance in between the nodes and $w_{i}$ being the inverse distance weight. Since a linear interpolation was chosen, value $p$ equals 1.

For example (see Eq. 7), if a remeshing is detected after increment $h=50$, a matrix based on the latched (surface) data is created, which contains the distances of each new and old node. With the linear search algorithm, the two nearest nodes $n_{1}$ and $n_{2}$ regarding the newly created node $n_{1}$ are detected. Then, it is possible to calculate, for example, the surface roughness $\mathrm{Sq}_{n_{l}}^{50+1}$ as follows (with $d_{1}=0.10 \mathrm{~mm}, \quad d_{2}=0.15 \mathrm{~mm}, \quad \mathrm{Sq}_{n_{1}}^{50}=4.2 \quad$ and $\left.\mathrm{Sq}_{n_{2}}^{50}=3.7\right)$ :

$$
\begin{aligned}
\mathrm{Sq}_{n_{l}}^{50+1} & =\frac{\sum \frac{\mathrm{Sq}_{i}^{50}}{d_{i}}}{\sum \frac{1}{d_{i}}}=\frac{\frac{\mathrm{Sq}_{n 1}^{50}}{d_{1}}+\frac{\mathrm{Sq}_{n 2}^{50}}{d_{2}}}{\frac{1}{d_{1}}+\frac{1}{d_{2}}} \\
& =\frac{\frac{4.2 \mu \mathrm{m}}{0.10 \mathrm{~mm}}+\frac{3.7 \mu \mathrm{m}}{0.15 \mathrm{~mm}}}{\frac{1}{0.10 \mathrm{~mm}}+\frac{1}{0.15 \mathrm{~mm}}}=4 \mu \mathrm{m}
\end{aligned}
$$

\section{Results}

\subsection{Sliding Compression Test}

In order to determine the model coefficients $p_{1}$ and $p_{2}$, sliding compression tests were performed at different loading levels, see Table 3. The loading levels, characterized by the normalized contact normal stress and surface enlargement, were determined with the help of FEA.

Figure 9a shows the friction coefficient $\mu$ in dependence of the load case and the lubrication system, while Fig. 9b presents the dependence of the mean square roughness Sq.

The measured friction coefficients show a qualitatively equal dependence in regard to both lubrication systems with a steady decline of the friction force. However, the friction coefficient for specimens lubricated with $\mathrm{MoS}_{2}$ is about $50 \%$ higher than for specimens lubricated with saltwax. 
Table 3 Comparison of loading cases

\begin{tabular}{lll}
\hline $\begin{array}{l}\text { Die force } \\
F(\mathrm{kN})\end{array}$ & $\begin{array}{l}\text { Normalized contact normal stress } \\
\sigma_{n} / k_{f}(-)\end{array}$ & $\begin{array}{l}\text { Surface enlargement } \\
\psi(-)\end{array}$ \\
\hline 200 & 0.98 & 1.55 \\
300 & 1.11 & 2.27 \\
450 & 1.28 & 4.16 \\
550 & 1.88 & 8.94 \\
\hline
\end{tabular}

In contrast, the leveling of the surface is highly dependent on the used lubrication system. While specimens lubricated with $\mathrm{MoS}_{2}$ show a gradual decline of surface roughness, specimens lubricated with salt-wax exhibit an increase in surface roughness for lower load cases. After this initial increase, the surface roughness for specimens lubricated with salt-wax also gradually declines for higher load cases $\left(\sigma_{n} / k_{f}>1.11\right)$. In addition, the variance of the surface roughness across the contact area is greater for this lubrication system.

Figure 10 depicts the surface evolution of the sliding compression specimens for the studied lubrication systems which were used to determine the surface roughness. While for loading cases around 1, some marks from the blasting process are still visible when using $\mathrm{MoS}_{2}$ as a lubricant, this cannot be observed in the case of salt-wax. With increasing loads, the surface morphology of specimens with salt-wax leads to the assumption that for low contact pressures the load is largely carried by the lubricant. For higher loads, an overall flattening of the surface asperities is observed, but still some rather large lubricant pockets remain. It can be assumed that under the given contact pressures, the salt-wax lubrication system exhibits fluidlike properties, while with a $\mathrm{MoS}_{2}$ lubrication, the surface asperities are in direct contact, thus resulting in a more smooth surface.

The parameters $p_{1}$ and $p_{2}$ for each lubrication system, determined with the help of a least squares fit of Eq. (4), as
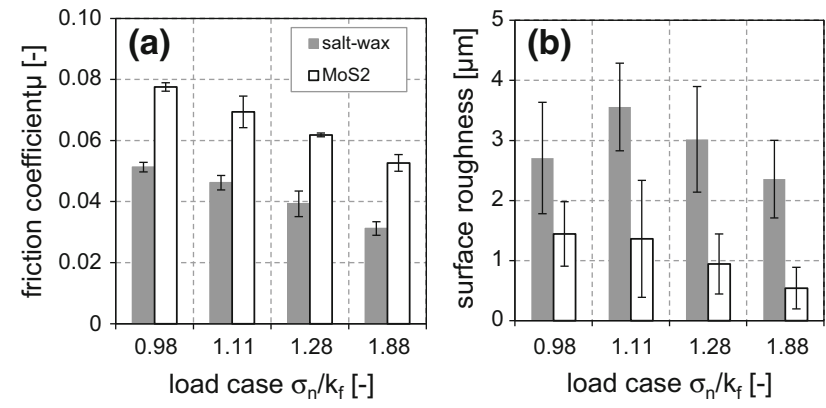

Fig. 9 Friction coefficient $\mu$ and roughness Sq

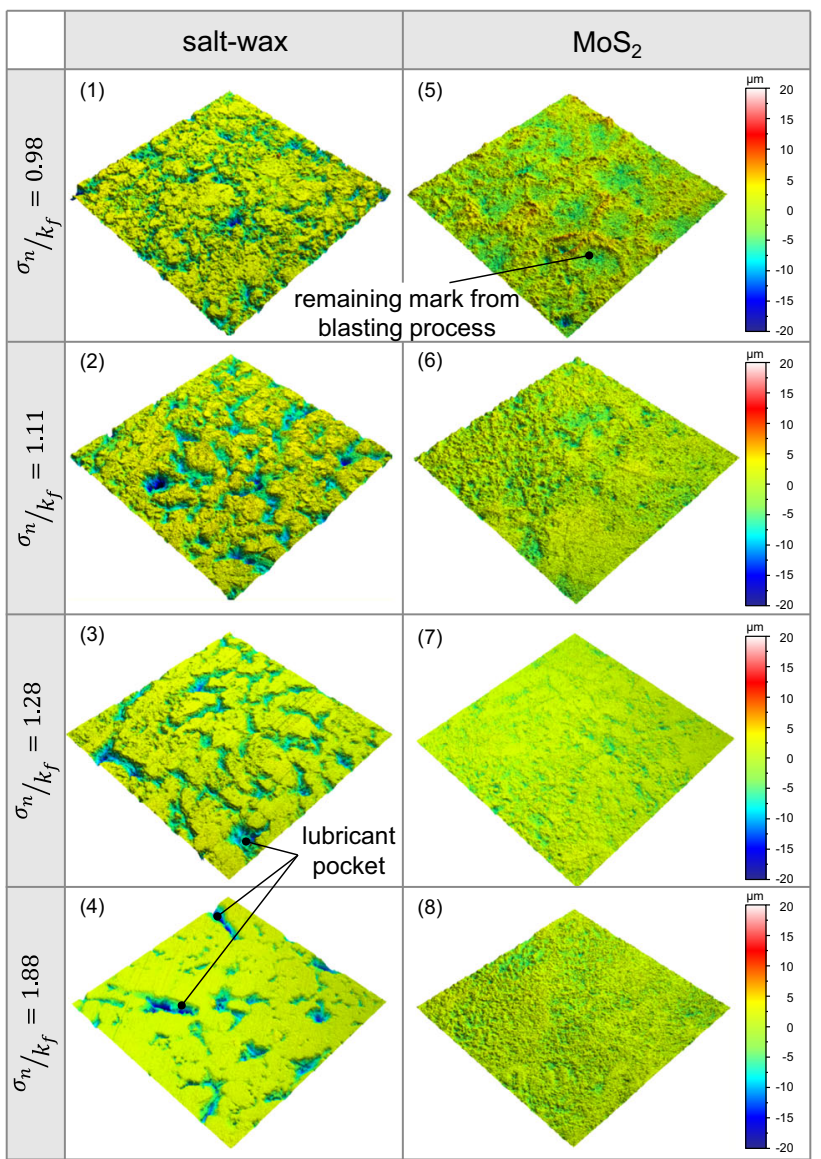

Fig. 10 Evolution of surface topography in dependence of load case and lubrication system

well as the lubrication system investigated by Stahlmann et al., are shown in Table 4.

By comparison, the influence of the surface enlargement is significantly lower for both investigated lubrication systems than for the originally studied lubrication system. In addition, it is remarked that the surface enlargement has a negative sign. This would lead to the conclusion that surface enlargement leads to a roughening of the surface. More likely, since the absolute value of $p_{1}$ is very small, another interpretation would be that the surface enlargement does not play a significant role toward calculating the surface roughness. This assumption is also supported by the fact that the absolute value of $p_{1}$ is at least one magnitude lower than the value $p_{2}$.

Table 4 Comparison of model parameters

\begin{tabular}{rrrr}
\hline & $\mathrm{MoS}_{2}$ & Salt-wax & {$[15]$} \\
\hline$p_{1}$ & -0.04 & -0.01 & 0.79 \\
$p_{2}$ & 0.63 & 0.31 & 0.26 \\
\hline
\end{tabular}




\subsection{Rod Extrusion Process}

The averaged force-displacement curves as well as the standard deviations of the rod extrusion process for both lubrication systems are shown in Fig. 11.

The maximum occurring load differs by about $9 \%$. In addition, it is also noticeable that the general trend of the force displacement curves differs. The force-displacement curve of the $\mathrm{MoS}_{2}$ lubrication system follows a linear downward trend after reaching the maximum force level. In contrast, the salt-wax-lubricated specimens exhibit a sharper fall of force after reaching the maximum level after which a linear trend is apparent. This is mainly due to the varying sticking friction coefficient of the lubrication systems. Also, the difference of deviations in between the series becomes obvious. The source of the high deviations for specimens lubricated with $\mathrm{MoS}_{2}$ lies in the difference in between the three test runs. These deviations could be a result of remaining lubricant within the die. Although the die was cleaned with acetone in between test runs, it proved difficult to clean all remaining lubricant.

The force-displacement curves were used to calibrate the FE-model of the rod extrusion process. The best fit for the $\mathrm{MoS}_{2}$ lubrication system was found for a friction

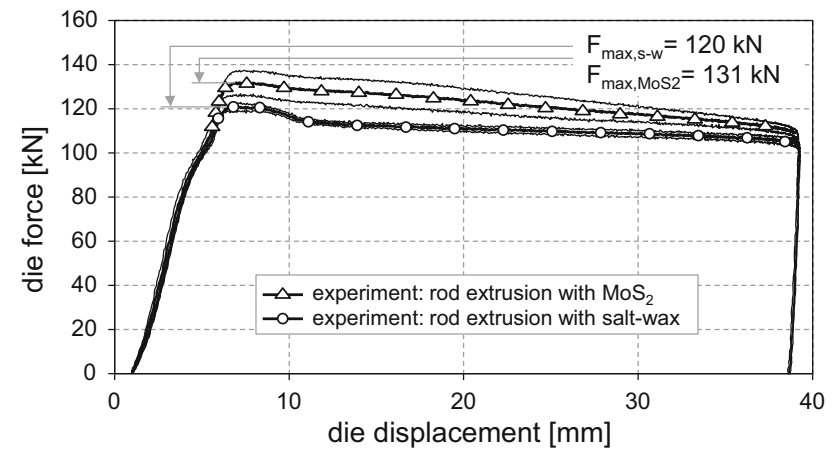

Fig. 11 Comparison of experimentally determined force-displacement curves

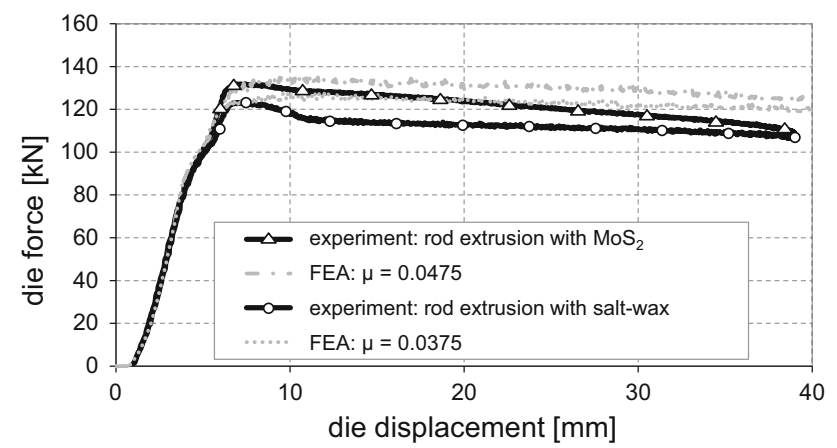

Fig. 12 Experimentally and numerically determined force-displacement curves coefficient $\mu_{\mathrm{MoS} 2}=0.0475$ and for the salt-wax lubrication system $\mu_{s-w}=0.0375$, see Fig. 12 .

Overall, the findings of the rod extrusion process are in agreement with the SCT when looking at the forming forces. The maximum registered forming forces for the rod extrusion process performed with $\mathrm{MoS}_{2}$ lie above the maximum forming forces of the salt-wax lubrication system. This coincides with the experimentally determined friction coefficients in the SCT, where the friction is lower for the salt-wax lubrication system compared to the $\mathrm{MoS}_{2}$ lubrication system for equal loads.

\subsection{Numerical Simulation}

Figure 13b, c depicts the resulting work pieces of the rod extrusion process as well as the path along the outline of the specimen. The surfaces of specimens extruded with a lubrication system consisting of salt-wax appear dull, while the surfaces of specimens with $\mathrm{MoS}_{2}$ have a shiny appearance. Measurements conducted with confocal white light microscopy, depicted in Fig. 13d, confirm that the surface of $\mathrm{MoS}_{2}$-lubricated specimens has a lower roughness, i.e., more smooth.
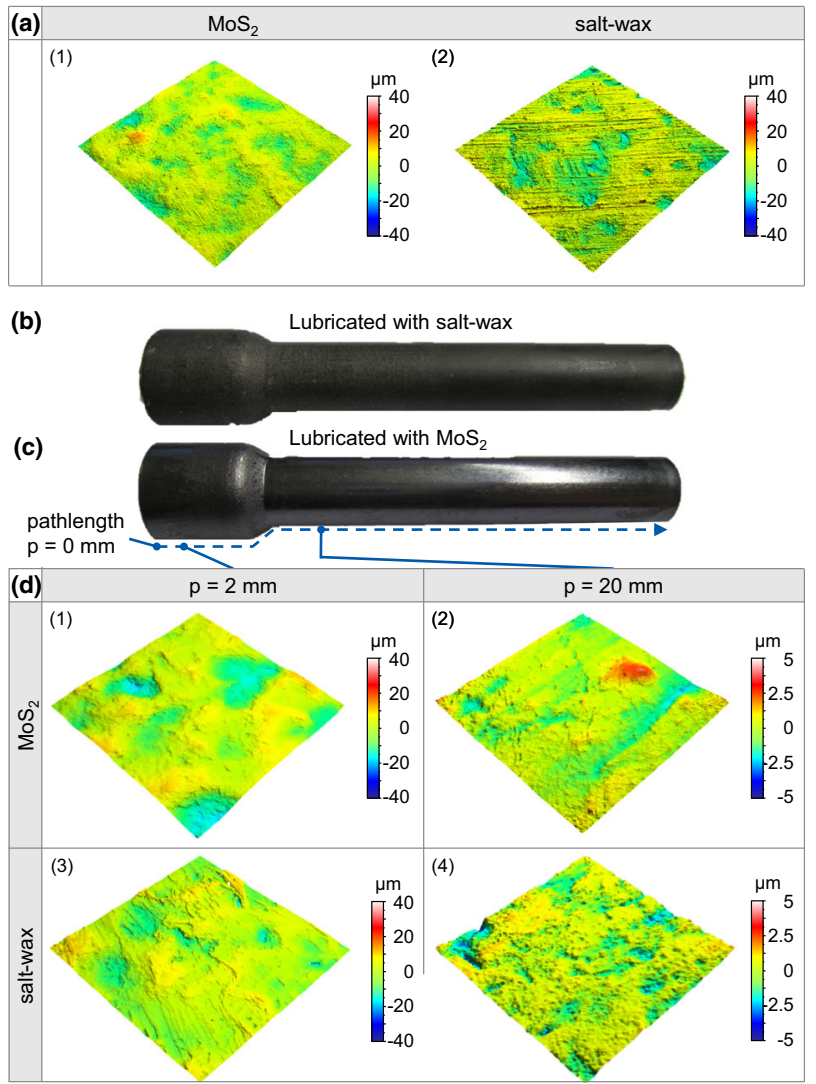

Fig. 13 Initial specimen surfaces (a), extruded rods (b, c) with corresponding surface roughness (d) 
The experimentally determined surface roughness and the numerically calculated surface roughness are compared in Fig. 14. The surface roughness is significantly reduced for specimens lubricated with both lubrication systems after the forming zone. While specimens with $\mathrm{MoS}_{2}$ have an average roughness of $\mathrm{Sq}=0.58 \pm 0.17 \mu \mathrm{m}$ after the forming zone, the roughness of specimens lubricated with salt-wax is higher with an average roughness $\mathrm{Sq}=1.05 \pm$ $0.28 \mu \mathrm{m}$

While the numerical prediction for both lubrication systems is accurate for work piece surfaces which have been formed (zone 3: Z3, see Fig. 15), the numerically predicted work piece roughness for the surface above the forming zone (zone 1: Z1) differs to a great extend in relation to the measured roughness. Within $Z 1$, the roughness is actually higher than the initially determined roughness of the lubricated specimen. It can be assumed that due to the low contact normal stress in zone 1, see Fig. 15, the surface asperities are not leveled. Excess lubricant, which adheres to the container, is transferred to the work piece during the forming process in $\mathrm{Z1}$, resulting in a higher roughness. This theory is supported by Fig. 13a, where it can be seen that the initially lubricated surfaces exhibit only few elevations of more than $10 \mu \mathrm{m}$ (color yellow), whereas the formed specimens in Z1, see Fig. 13d $(1,3)$, exhibit numerous elevations of $10 \mu \mathrm{m}$ and higher which can be ascribed to the lubricant. Due to a possible more homogenous consistence of $\mathrm{MoS}_{2}$, the increase in
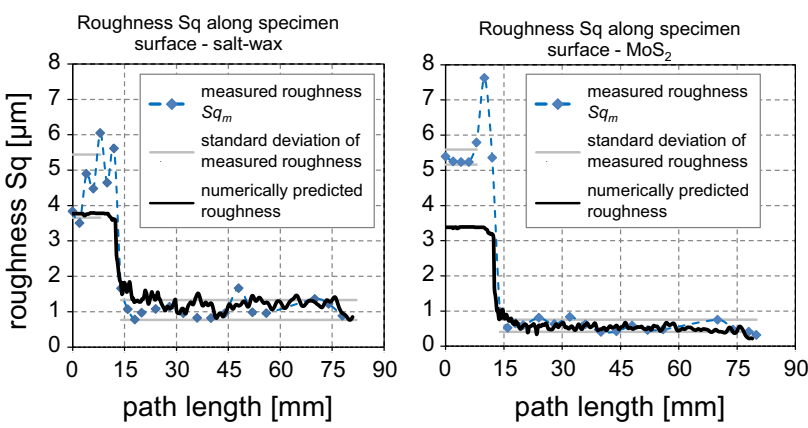

Fig. 14 Comparison of experimentally and numerically determined surface roughness

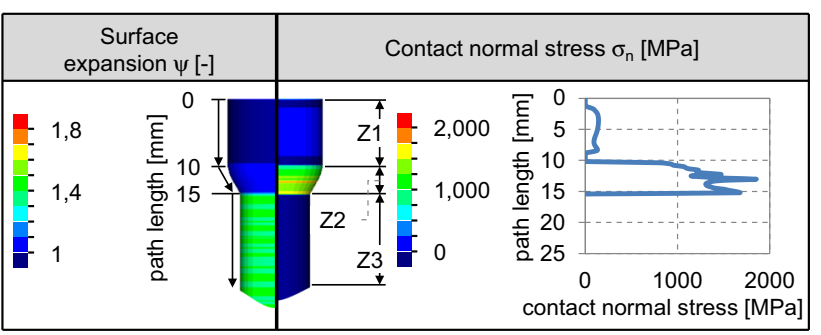

Fig. 15 Comparison of tribological loads roughness is more evenly distributed compared to saltwax. Crossing over into zone 2 (Z2), the contact normal stress $\sigma_{n}$ increases significantly while the surface expansion $\psi$ increases only slightly. Both causes result in the leveling of the surface asperities. The peak of roughness, seen in Fig. 14 for $\mathrm{MoS}_{2}$, can be attributed to the incline of surface enlargement in combination with a loss of contact crossing over from Z1 into Z2, as observed in Fig. 15, which leads to a high localized roughness due to unbounded forming. The lower maximum roughness for lubrication with saltwax in comparison with $\mathrm{MoS}_{2}$ is most likely a result of the transferal of lubricant into the forming shoulder, thus averting an unbounded forming.

In conclusion, Fig. 14 depicts that the proposed model shows a good accordance with the experimentally obtained measurements. Both predictions lie within the error bars gathered by measurement.

\section{Conclusion and Outlook}

It was demonstrated that the proposed surface evolution model in [15] is applicable to common surface preparation techniques and lubrication systems as used in the cold forming industry. The numerical prediction of the surface roughness shows good agreement with the experimental results.

For future research, the surface evolution model can be used to calculate a local friction coefficient depending on tribological loads as well as the surface roughness. This is necessary, because, as can be seen in Fig. 12, the deviation of numerically and experimentally determined force-displacement curves is still rather large. In order to minimize this deviation, the modeling of friction has to be improved. Next to modeling the slipping friction, a consideration of the lubricant specific sticking friction is necessary to ensure a correct depiction of stresses within the work piece.

Acknowledgments The authors would like to thank the German Research Foundation (DFG) for funding this research project.

Open Access This article is distributed under the terms of the Creative Commons Attribution 4.0 International License (http:// creativecommons.org/licenses/by/4.0/), which permits unrestricted use, distribution, and reproduction in any medium, provided you give appropriate credit to the original author(s) and the source, provide a link to the Creative Commons license, and indicate if changes were made.

\section{References}

1. Joun, M.S., Moon, H.G., Choi, I.S., Lee, M.C., Jun, B.Y.: Effects of friction laws on metal forming processes. Tribol. Int. 42(2), 311-319 (2009) 
2. Bay, N.: The state of the art in cold forging lubrication. J. Mater. Process. Technol. 46, 19-40 (1994)

3. Bay, N., Eriksen, M., Tan, X., Wibom, O.: A friction model for cold forging of aluminum, steel and stainless steel provided with conversion coating and solid film lubricant. CIRP Ann. Manuf. Technol. 60(1), 303-306 (2011)

4. Groche, P., Müller, C., Stahlmann, J., Zang, S.: Mechanical conditions in bulk metal forming tribometers-part one. Tribol. Int. 62, 223-231 (2013)

5. Müller, C., Keller, M., Groche, P.: Independent investigation of the contact normal stress and the surface enlargement with the sliding compression test. In: Proceedings of the 16th International Conference on Advances in Material and Processing. Technologies, Taipei, Taiwan (2013)

6. Kudo, H.: A note on the role of microscopically trapped lubricant at the tool-work interface. Int. J. Mech. Sci. 7(5), 383-388 (1965)

7. Hu, Z.M., Dean, T.A.: A study of surface topography, friction and lubricants in metalforming. Int. J. Mach. Tools Manuf. 40, 1637-1649 (2000)

8. Jung, K.H., Lee, H.C., Kang, S.H., Im, Y.T.: Effect of surface roughness on friction in cold forging. J. Achiev. Mater. Manuf. Eng. 31(2), 327-334 (2008)

9. Cristino, V.A.M., Rosa, P.A.R., Martins, P.A.F.: Surface roughness and material strength of tribo-pairs in ring compression tests. Tribol. Int. 44(2), 134-143 (2001)

10. Sahin, M., Çetinarslan, C.S., Akata, H.E.: Effect of surface roughness on friction coefficients during upsetting processes for different materials. Mater. Des. 28(2), 633-640 (2007)
11. Greenwood, J.A., Williamson, J.B.P.: Contact of nominally flat surfaces. Proc. R. Soc. A Math. Phys. Eng. Sci. 295(1442), 300-319 (1966)

12. Hol, J., Meinders, V.T., de Rooij, M.B., van den Boogaard, A.H.: Multi-scale friction modeling for sheet metal forming: the boundary lubrication regime. Tribol. Int. 81(1), 112-128 (2015)

13. Korzekwa, D.A., Dawson, P.R., Wilson, W.R.D.: Surface asperity deformation during sheet forming. Int. J. Mech. Sci. 34(7), 521-539 (1992)

14. Kießling, A., Bartel, D., Deters, L.: Simulation der Festkörperreibung von rauen Oberflächen mittels FEM. In: Gesellschaft für Tribologie, 54. Tribologie-Fachtagung, vol. 2, 2/1-2/14 (2013)

15. Stahlmann, J., Nicodemus, E.R., Sharma, S.C., Groche, P.: Surface roughness evolution in FEA simulations of bulk metal forming processes. Wear 288, 78-87 (2012)

16. German Cold Forging Group (GCFG): Information sheet for determination of flow stress curves, German (2008)

17. Hemyari, D.: Methode zur Ermittlung von Konstitutivmodellen für Reibvorgänge in der Massivumformung bei erhöhten Temperaturen, Dissertation, Technische Universitaet Darmstadt, Shaker Verlag, Aachen, German (1999)

18. Groche, P., Stahlmann, J., Hartel, J., Köhler, M.: Hydrodynamic effects of macroscopic deterministic surface structures in cold forging processes. Tribol. Int. 42(8), 1173-1179 (2009) 